\title{
Padrão de metadados no domínio museológico
}

\author{
Fábio Rogério Batista Lima
}

\begin{abstract}
Mestre em Ciência da Informação e doutorando no Programa de Pós-Graduação em Ciência da Informação da Universidade Estadual Paulista Júlio de Mesquita Filho - Campus de Marília
\end{abstract}

Plácida Leopoldina V. A. C. Santos

Livre docente em catalogação e doutora em letra pela Universidade de São Paulo, Brasil. Docente do Programa de Pós-Graduação em Ciência da Informação da Universidade Estadual Paulista Júlio de Mesquita Filho - Campus de Marília

José Eduardo Santarém Segundo

Doutor em Ciência da Informação e docente do Programa de Pós-Graduação em Ciência da Informação da UNESP/Marília e docente e coordenador do Curso de Graduação em Ciências da Informação e da Documentação e Biblioteconomia, da Faculdade de Filosofia, Ciências e Letras de Ribeirão Preto

http://dx.doi.org/10.1590/1981-5344/2639

É no ambiente Web que os museus estão tornando acessíveis, de forma digital, grande parte das informações sobre seu acervo. No entanto, essas informações estão sendo disponibilizadas de forma não estruturada. Isso dificulta a troca de dados, a comunicação e a interoperabilidade entre sistemas de museus, que necessitam de padrões de metadados adequados para gerenciar e disponibilizar informações vinculadas a seus acervos. A questão de investigação é: como os padrões de metadados podem contribuir para os museus de arte? A pesquisa apresenta abordagem qualitativa, é de caráter teórico de nível descritivo e exploratório. O resultado da análise demonstrou que nos museus de arte brasileiros não são utilizados padrões de metadados, e tampouco há literatura nacional que aborde esse tema. Contudo, em alguns países utiliza-se alguns padrões destinados ao universo museológico, dentre os quais o VRA Core versão 4.0, que para a catalogação de obras de arte no ambiente digital parece ser o mais adequado. 
Palavras-chave: Padrão de metadados para obras de arte; VRA Core; Acervo digital; Museu no ambiente Web.

\section{Standard metadata in museological domain}

It is in the Web environment that museums are making much of the information about their collection accessible in digital form. However, this information is being made available in an unstructured form. It hampers the exchange of data, communication and interoperability between museum systems that require suitable metadata standards to manage and provide information linked to their collections. The research question is: how can metadata standards contribute to art museums? The research presents a qualitative approach is theoretical character of descriptive and exploratory level. The result of the analysis showed that the metadata standards are not being used in Brazilian art museums, and there are not even national literature for dealing with this issue. However, in some countries is used some standards for the museological universe, of which the VRA Core version 4.0, which for cataloging works of art in the digital environment seems to be the most appropriate.

Keywords: Metadata standard for works of art; VRA Core; Digital collection; Museum in the Web environment.

Recebido em 27.01.2016 Aceito em 25.08.2016

\section{Introdução}

A era digital trouxe consigo um mundo mais dinâmico, interativo e informativo. A Internet, ou também chamada de rede mundial de computadores, através do protocolo de comunicação TCP/IP ${ }^{1}$, permitiu acesso a variadas fontes e recursos informacionais. Com o auxílio de aparatos tecnológicos cada vez mais sofisticados e de tecnologias de informação e comunicação (TIC) somadas à evolução do ambiente Web, tornou-se possível dar um novo direcionamento para as formas tradicionais de acesso, recuperação, armazenamento e gestão da informação.

\footnotetext{
${ }^{1}$ TCP/IP - Transmission Control Protocol/Internet Protocol.
} 
Ambientes onde há interação de informação, a exemplo, instituições responsáveis por patrimônios culturais, como os museus, os quais "trabalham com a organização, o tratamento, o armazenamento, a recuperação e a disseminação da informação produzida a partir de suas coleções" (YASSUDA, 2009, p. 15) estão sendo afetados por aparatos tecnológicos e ambientes digitais de informação e obrigados a repensar uma nova forma de produção, gerenciamento e recuperação da informação em seu acervo.

É no ambiente Web que está sendo disponibilizada, de forma digital, uma exponencial quantidade de recursos informacionais, nos mais variados tipos de fontes. No entanto, essas informações estão sendo disponibilizadas de forma não estruturada, dificultando a troca de dados e a comunicação entre sistemas e plataformas.

No entanto, para que existam programas que coletem conteúdos em diversas fontes, troquem resultados, relacionando-se com outros programas, bem como possibilitem o processamento adequado da informação, é necessário que as instituições, neste caso especifico os museus, que atualmente possuem algum "tipo de recursos que incluem imagens digitais estáticas, textos digitais, arquivos digitais de áudio e arquivos de imagem digitais em movimento" (MILLER, 2004, p. 6, tradução nossa), adiram a estruturas padronizadas do conteúdo informacional, que será representado pelo conjunto de atributos de sua coleção. Neste sentido, é necessário que elas trabalhem com padrões específicos de metadados.

O estudo em questão partiu da problemática: como os padrões de metadados podem contribuir para os museus de arte? Este estudo é de caráter teórico de nível descritivo e exploratório. A pesquisa apresenta abordagem qualitativa, tendo como base a observação sistemática da bibliografia coletada utilizando o suporte teórico da Ciência da Informação nas questões relacionadas a metadados no universo museológico. Teve como objetivo geral analisar os padrões de metadados utilizados na descrição de objetos de arte e, específico, analisar qual dentre eles seria o ideal para se trabalhar com a catalogação desses objetos.

\section{Metadados}

Metadados é um termo genérico que abrange uma ampla variedade de tipos específicos de informações, as quais são criadas ou capturadas sob vários tipos de recursos informacionais. O termo é usado frequentemente para referir-se às informações legíveis por máquinas, outras vezes para referir-se aos registros que descrevem recursos eletrônicos. Na comunidade biblioteconômica, por exemplo, o termo é usado para se referir a qualquer esquema de descrição de recurso, aplicado a qualquer tipo de objeto, sendo ele digital ou não.

Os metadados são essenciais para o entendimento do recurso armazenado, pois descrevem informações semânticas e sintáticas sobre o recurso, podendo ser comparados a um sistema de rotulagem, com 0 
objetivo de mostrar como, quando e por quem o recurso foi armazenado e como está formatado.

São dados descritivos que podem informar sobre autor, título, data, publicação, palavras-chave, descrição física, entre outros, nos mais variados tipos de recurso, como em arquivos de áudio, conjunto de dados científicos, imagem digital, catálogos de museu, livros etc., como mostra o quadro 1

Quadro 1 - Metadados de um livro

LC Control No.: 2008015176

LCCN Permalink: http//iccn.loc.gov/2008015176

Type of Material: Book (Print, Microform, Electronic, etc)

Personal Name: Zeng, Marcia Lei, 1956 -

Main Title: Metadata / Marcia Lei Zeng and Jian Qin.

Published/Created: New York : Neal-Schuman Publisher, c2008.

Related Names: Qin, Jian, 1956-

Description: $x v i i, 365$ p. : ill. $23 \mathrm{~cm}$.

ISBN: 9781555706357 (pbk. : alk. paper)

1555706355 (pbk. : alk. paper)

Contents: Introduction - Current standards - Schemas : structure and semantics - Schemas : syntax - metadata records - Metadata services - Metadata quality measurement and improvement.

Notes: Includes bibliographical references (p.327-357) and index.

Subject: Metadata

LC Classification: Z666.7 .Z45 2008

Dewey Class No. : 025.322

Source Library of Congress Online Catalog: HTTP//catalog.loc.gov/.

Fonte: MILLER (2004, p. 4).

Os metadados "são dados ou informações que permitem às pessoas exercerem determinadas funções em relação aos recursos de informação a que os metadados se referem [...]" (MILLER, 2004, p.1, tradução nossa).

A publicação "Understanding Metadata" da NATIONAL INFORMATION STANDARDS ORGANIZATION (2004) define metadados como

informação estruturada que descreve, explica, localiza, ou ainda, possibilita que um recurso informacional seja fácil de recuperar, de usar ou gerenciar. O termo metadados, frequentemente, designa dados sobre dados ou informação sobre informação. 
Outros autores, como Alves (2010, p. 47), os descrevem como:

Atributos que representam uma entidade (objeto do mundo real) em um sistema de informação. Em outras palavras, são elementos descritivos ou atributos referenciais codificados que representam características próprias ou atribuídas às entidades; são ainda dados que descrevem outros dados em um sistema de informação, com o intuito de identificar de forma única uma entidade (recurso informacional) para posterior recuperação.

Os metadados facilitam a forma de entendermos a relação entre as informações e os dados fornecidos nas mais variadas formas e contextos. Para isso, existem tipos diferentes de padrões de metadados, alguns específicos para uma determinada área, outros gerais, que servem para variados fins, como será visto na próxima seção.

\subsection{Padrões de metadados}

O termo padrão de metadados (standard Metadata), também chamado de formatos de metadados, é entendido, dentre um vasto leque de formalidades, como os padrões emitidos por agências de normas e outras organizações, tais como: International Organization for Standardization (ISO), the American National Standards Institute (ANSI), the Dublin Core Metadata Iniciative (DCMI), the Library of Congress (LC), the Getty Research, the Wide Web Consortium (W3C), entre outros.

Como exemplo de padrões de metadados, temos o Machine Readable Cataloging (MARC) usado para recursos Catalográficos, o Metadado Brasileiro para Teses e Dissertações (MTD-BR), Categories for the Description of Works of Art (CDWA), Computer interchange of museum information (CIMI), Dublin Core (DC), The Visual Resources Association (VRA Core), dentre outros.

Atualmente, há grande variedade de tipos de padrões de metadados, os quais são usados em diversos contextos, como em empresas, pesquisas, educação, saúde, leis, negócios, comércio na Internet, bem como em instituições que trabalham com objetos de arte. Alguns tipos de metadados têm como função gerenciar, administrar ou preservar recursos ou estruturas internas de complexos objetos digitais, a exemplo o quadro 2. 
Quadro 2 - Tipologia de metadados

\begin{tabular}{|c|c|c|}
\hline Tipo & Definição & Exemplo \\
\hline Administrativo & $\begin{array}{c}\text { metadados usados no gerenciamento e } \\
\text { na administração de recursos } \\
\text { informacionais. }\end{array}$ & $\begin{array}{l}\text { - Aquisição de informação; } \\
\text { - Acompanhamento de direitos e de } \\
\text { reprodução; } \\
\text { - Documentação de requisitos para o } \\
\text { acesso legal; } \\
\text { - Localização de informação; } \\
\text { - Critérios de seleção para digitalização; } \\
\text { - Controle de versão e diferenciação entre } \\
\text { objetos de informação similares; } \\
\text { - Pistas à auditoria criadas por sistemas } \\
\text { de manutencão de registros. }\end{array}$ \\
\hline Descritivo & $\begin{array}{l}\text { metadados usados para descrever ou } \\
\text { identificar recursos informacionais }\end{array}$ & $\begin{array}{l}\text { - Ferramentas para localizar; } \\
\text { - Índices especializados; } \\
\text { - Relacionamentos de hyperlinks entre } \\
\text { recursos; } \\
\text { - Anotações dos usuários; } \\
\text { - Metadados para sistemas de } \\
\text { manutenção de registros gerados, pelos } \\
\text { criadores dos registros. }\end{array}$ \\
\hline de Preservação & $\begin{array}{l}\text { metadados relacionados com o } \\
\text { gerenciamento de preservação dos } \\
\text { recursos informacionais }\end{array}$ & $\begin{array}{l}\text { - Documentação da condição física dos } \\
\text { recursos; } \\
\text { - Documentação das ações realizadas } \\
\text { para preservar as versões digitais e } \\
\text { físicas dos recursos, por exemplo, } \\
\text { atualização e migração de dados. }\end{array}$ \\
\hline Técnicos & $\begin{array}{l}\text { metadados relacionados ao } \\
\text { funcionamento de um sistema ou ao } \\
\text { comportamento dos metadados }\end{array}$ & $\begin{array}{l}\text { - informações sobre hardware e software; } \\
\text { - autenticidade e segurança dos dados } \\
\text { etc. }\end{array}$ \\
\hline de Uso & $\begin{array}{l}\text { metadados relacionados com o nível e } \\
\text { tipo de uso dos recursos informacionais }\end{array}$ & $\begin{array}{l}\text { - Registros exibidos; } \\
\text { - Acompanhamento de uso e de usuários; } \\
\text { - Reúso de conteúdo e informações em } \\
\text { múltiplas versões. }\end{array}$ \\
\hline
\end{tabular}

Fonte: BACA (1998).

Para Miller (2004), na literatura sobre metadados de patrimônio cultural, em sua maior parte, os metadados são caracterizados e divididos em três categorias: metadados administrativos, metadados estruturais e metadados descritivos.

Há especialistas que, além dessas categorias mencionadas, incluem metadados de preservação, metadados técnicos e metadados de uso, como demonstrado no quadro 2.

O tipo mais frequente de metadados destinados a objetos de arte em ambiente digital são os metadados descritivos, pois eles fornecem acesso aos conteúdos de uma coleção digital. Sem este tipo de metadados, as coleções seriam inúteis, pois os usuários não teriam como encontrar e identificar o objeto dentro da coleção.

A documentação museológica, neste sentido, destina-se ao tratamento da informação, desde a entrada do objeto no museu até a exposição. Isso faz parte de um dos aspectos da gestão dos museus. O museu é uma instituição complexa por concepção e gestão, que responde às mudanças culturais, políticas e sociais das comunidades onde se encontra (CRIPPA, 2010, p. 32). 
Neste processo, "estão envolvidas tarefas direcionadas à coleta, armazenamento, tratamento, organização, disseminação e recuperação da informação" (YASSUDA, 2009, p. 19).

A documentação de museus, segundo Ferrez (1991, p. 1), "[...] é o conjunto de informações sobre cada um dos seus itens e, por conseguinte, a representação destes por meio da palavra e da imagem (fotografia)." A documentação museológica serve como um sistema de recuperação da informação, que transforma as coleções dos museus de fontes de informação em fontes de pesquisa, servindo para transmissão do conhecimento.

Segundo Yassuda (2009, p. 36), os métodos de documentação de museu, hoje,

[...] continuam em processo de estruturação, adaptando-se às tecnologias vigentes no mundo globalizado. A tendência à interoperabilidade de informação é crescente e se mostra como um grande desafio para os museus da contemporaneidade.

A "maioria dos procedimentos de criação de dados é conduzida por meio de padrões, normas e instruções bem consolidadas, incluindo aquelas para estruturação de dados (por exemplo, esquemas de metadados), conteúdo de dados (por exemplo, princípios e regras), $[\ldots]^{\prime \prime}$ (ZENG, 2013, p. 95).

A utilização de padrões de metadados nas coleções de museu facilita a troca de dados entre museus que utilizam o mesmo padrão, auxiliam a recuperação automática da informação e promovem a consistência nos bancos de dados, tornando mais fácil o compartilhamento de informações entre eles, pois tanto os padrões de conteúdos, padrões externos, códigos e regras são determinantes não só para a padronização da sintaxe dos metadados mas também para a padronização nos valores da representação (ZENG; QIN, 2008).

Atualmente, existem iniciativas e empresas que se preocupam e discutem soluções para a elaboração de padrões de metadados para objetos de arte, especificamente para o ambiente Web.

O Canadian Heritage Information Network $(\mathrm{CHIN})^{2}$, juntamente com alguns museus do Canadá, trabalha na intenção de armazenar e apresentar o conteúdo digital dos acervos para o público.

Outras iniciativas são: o projeto Europeana ${ }^{3}$, criado em meados de $2005 \mathrm{com}$ a finalidade de tornar acessível a todos o patrimônio cultural da Europa por meio da criação de uma biblioteca virtual em cooperação com as bibliotecas nacionais e outros organismos culturais dos EstadosMembros. Reúne as principais associações de bibliotecas, arquivos, museus, arquivos audiovisuais e instituições culturais da Europa.

${ }^{2}$ CHIN. Disponível em: <http://www.chin.gc.ca/>. Acesso em: 27 jul. 2016. O padrão CHIN é baseado no Dublin Core e inclui controle de vocabulário para projetos de aprendizagem.

Disponível em: <https://ec.europa.eu/digital-single-market/en/europeana-european-digital-library-all>. Acesso em: 27 jul. 2016. 
A Europeana está instalada na biblioteca nacional neerlandesa, a Koninklijke Bibliotheek e já recebeu contribuições de mais de 2.200 organizações culturais de toda a Europa. Utiliza o Europeana Data Model (EDM),

O EDM é um modelo de dados teórico que permite que os dados sejam apresentados de diferentes maneiras de acordo com as práticas dos vários domínios que contribuem para a Europeana (EUROPEANA, 2014, não paginado, tradução nossa).

É um esquema de metadados cujos elementos são comuns a todas as comunidades de fornecedores de dados e que permite o mapeamento dos diversos conjuntos de dados para uma representação uniforme dos dados (ISAAC, 2012).

E também o Museum Initiative for Digital Information Interchange Standards (MIDIIS) ${ }^{4}$, que possui, como uma de suas metas, buscar entender o universo das informações de coleções museológicas. Para isso, busca saber dos museus quais são os tipos de informações que eles utilizam na descrição e representação de suas coleções. A intenção é criar metadados para promover a interoperabilidade e a troca de informações entre os museus.

O MIDIIS é apoiado pelo Consórcio para Intercâmbio de Informação de Museu (CIMI) ${ }^{5}$, e envolve o Dublin Core como formato de metadados. Dessa forma, o CIMI

desenvolveu o XML Schema para descrição de objetos de museus. Este programa foi baseado no SPECTRUM e denomina-se CIMI XML Schema. Ele permite uma rica descrição da informação relativa aos objetos de museus, incluindo informações associadas a pessoas, lugares e eventos acerca da história dos objetos, bem como informação sobre o gerenciamento e uso (YASSUDA, 2009, p. 37).

O projeto tem a preocupação de saber como os museus podem prover, efetivamente, 0 acesso às informações criadas por eles para representar suas coleções que são mantidas em formato digital, como: informações sobre coleções, publicações, eventos, atividades educacionais e pessoas, bem como servir para a compreensão das diferentes formas estruturais dos museus, a fim de testar a linguagem de marcação XML (eXtensible Markup Language), como sintaxe para representar a identificação semântica das informações e desenvolver uma DTD (Document Type Definition) para representar diferentes tipos de informações.

\footnotetext{
${ }^{4}$ Disponível em: <http://www.cimi.org>. Acesso em: 27 jul. 2016.

${ }^{5}$ Disponível em: <http://www.cimi.org>. Acesso em: 27 jul. 2016.
} 


\section{2 Padrões de metadados para objetos de museus}

Há algum tempo, os museus, de modo geral, não se preocupavam nem tinham uma tradição em manter algum tipo de padronização na forma de descrição de seus recursos, a qual era feita basicamente escrevendo-se ou datilografando-se os metadados dos registros. No entanto, com o surgimento da representação de objetos digitais nas coleções digitais disponibilizadas on-line, mudou o cenário museológico. Assim, foi "a partir do trabalho de autores como Alma S. Wittlin, Francis Henry Taylor $[\ldots]$, além do impulso dado pela formação da Museum Documentation Association ${ }^{6}$, que se desenhou um campo de estudos em torno da documentação museológica" (ARAújO, 2014, p. 80-81).

Atualmente os museus têm interesse maior pela padronização e compartilhamento de metadados, além de verem a importância da atualização quanto à forma de registrar seus documentos.

Existe ontologias de referência, como a ISO 21127: 2014 ${ }^{7}$, que estabelece as diretrizes para a troca de informações entre instituições de patrimônio cultural bem como alguns tipos de padrões de metadados que foram desenvolvidos para trabalhar especificamente com patrimônios culturais, como obras de arte, arquitetura e imagens desses objetos, a exemplo: Categories for the Description of Works of Art (CDWA) - e o The Visual Resources Association (VRA) Core. Esses padrões se propõem assegurar que informações importantes sejam registradas, auxiliam a recuperação automática de informação, promovem a consistência entre bancos de dados, bem como facilitam a migração de dados para sistemas novos.

O guia utilizado por esses padrões para descrever o conteúdo das obras e suas imagens é o Cataloging Cultural Objects (CCO) ${ }^{8}$, um padrão de conteúdo concebido para aqueles que trabalham com a descrição de obras de arte, arquitetura e artefatos culturais. São utilizados também padrões de valores como: Art \& Architecture Thesaurus (AAT), Union List of Artist Names (ULAN), entre outros. Ambos fazem parte do Getty Foundation e estão disponibilizados no site ${ }^{9}$.

No panorama brasileiro, o que ocorre tanto em arquivos quanto em museus é a tendência de essas instituições quererem resolver seus problemas usando padrões da biblioteconomia, pois nesta última os padrões de metadados estão mais consolidados quando comparados aos padrões utilizados em arquivos e museus (COSTA; ALMEIDA, 2010; BEVILACQUA, 2014).

Pode-se partir da experiência já consolidada da biblioteconomia, mas com cuidado para não deixar de lado as especificidades dessas outras áreas, as quais demandam uma atenção especial devido às nuances de seus objetos e, também, para que não ocorra uma simplificação exagerada nos metadados.

\footnotetext{
${ }^{6}$ Disponível em: <http://www.museumsassociation.org/home>. Acesso em: 27 jul. 2016.

Disponível em: <http://www.iso.org/iso/home/store/catalogue_tc/catalogue_detail.htm?csnumber=57832>. Acesso em: 27 jul. 2016.

${ }^{8} \mathrm{CCO}$ é um padrão de conteúdo de dados publicado em 2006, patrocinado pela VRA, e publicado pela American Library Association (ALA). O projeto foi, em grande parte, financiado pela Fundação Getty.

${ }^{9}$ Disponível em: <http://www.getty.edu/research/tools/vocabularies/ulan/index.html>. Acesso em: 27 jul. 2016.
} 
Um dos fatores que impede a utilização de padrões de metadados nos museus de arte brasileiros é o fato de que a maioria dos padrões que existem e que estão em estágios avançados em países mais desenvolvidos não estão traduzidos para o português. Ou seja, há uma barreira de linguagem e também, pelo fato de os processos de informatização nos museus do Brasil serem de baixa qualidade, devido à ausência de padrões estabelecidos e aos baixos índices de duração dos mesmos, que não superam os dois anos de utilização, até serem substituídos por um novo projeto Bevilacqua (2014).

Existem, como dito anteriormente, algumas iniciativas para acervos museológicos, como por exemplo o CHIN, MIDIIS e a Europeana, mas aqui no Brasil nada que aponte para uma solução. Tudo é muito estanque ainda. No entanto, cada área procura a sua solução - bibliotecas, arquivos e museus. A "área museológica, por não ter padrões de descrição consolidados, tem apresentado soluções particulares de sistemas de gestão de acervos" (MARCONDES, 2013, p. 13). Contudo, por que o fato é tão crucial para o museu? Porque o museu é uma das únicas instituições que tem os três acervos (bibliográfico, arquivístico e museológico) convivendo e disponíveis no mesmo espaço.

Dessa forma, os museus constituem um espaço muito interessante que sempre apresenta uma demanda de integração de sistemas e recuperação da informação. No entanto, por diversos motivos, em especial "pelo desenvolvimento independente desses sistemas, pelos diferentes padrões de descrição e representação de objetos nas áreas arquivistica, bibliográfica e museológica, estes sistemas hoje não são interoperáveis" (MARCONDES, 2013, p. 11).

Outro problema também relacionado a esses anteriores é a ausência de fornecedores qualificados. Talvez na Europa já exista um mercado com empresas fornecendo softwares para gestão de acervos museológicos. No Brasil, a maioria dos softwares foi adaptada de biblioteca para museus. Com a abertura do mercado, existem empresas especializadas, as quais vêm com esses padrões todos baseados no CIDOC e no Spectrum. É outra realidade que se aproxima.

Dessa forma, a tendência de usar metadados ainda é aquela de utilizar padrões já consolidados da biblioteconomia. Pois, segundo Marcondes (2013, p. 12),

A evolução histórica do desenvolvimento e amadurecimento dos padrões de descrição de informações em arquivos, bibliotecas e museus se deu em momentos distintos. O AACR2 - Código de Catalogação Anglo Americano 2a edição - e os experimentos com o padrão MARC de catalogação bibliográfica remontam à década de 60. A norma geral de descrição arquivística ISAD $(G)$ foi adotada pelo Conselho Internacional de Arquivos na década de 90. A área museológica não tem um padrão de descrição, mas sim um modelo de referencia, o CIDOC-CRM. Enquanto que o MARC é um padrão de fato para intercâmbio de dados bibliográficos, a norma ISAD(G) e o modelo CIDOC-CRM são diretrizes ou recomendações gerais.

Existem ferramentas especificas para se trabalhar com objetos culturais e artísticos, como o CDWA, o CCO, etc. Contudo, não abrangem todos os campos necessários. A tendência agora, é a busca de interoperabilidade entre esses modelos. 
Existe uma iniciativa que busca esta interoperabilidade e que surgiu como um grupo de trabalho dentro do CIDOC/ICOM, o Documentation Standards Working Group (DSWG) há mais de dez anos, chamado de Conceptual Reference Model (CRM). A interoperabilidade, neste sentido, "diz respeito à capacidade de sistemas diferentes trocarem e utilizarem dados gerados por outros sistemas" (MARCONDES, 2013, p. 10).

O que torna este modelo interessante é o fato de ser mais abrangente do que os demais, pois ele está focado não só em mapear os possíveis metadados de objetos, mas sim mapear as relações entre eles.

O CRM CIDOC se destina a promover uma compreensão partilhada de informações sobre o patrimônio cultural, fornecendo uma estrutura semântica comum e extensível de modo que qualquer informação sobre herança cultural possa ser mapeada. Destina-se a ser uma linguagem comum para especialistas em domínio e implementadores para formular requisitos para sistemas de informação e para servir como um guia para a boa prática de modelagem conceitual. Desta forma, ele pode fornecer a "cola semântica" necessária para mediar entre as diferentes fontes de informação de patrimônio cultural, como a publicada por museus, bibliotecas e arquivos (INTERNATIONAL COUNCIL OF MUSEUM/ CIDOC, 2014, não paginado, tradução nossa)

Este modelo é, de certa forma, um desdobramento para arquivos, porque ele vai além da questão do item, do objeto enquanto unidade intrínseca. Ele foca a relação dos objetos com o produtor, e para museu e arquivo há uma demanda muito maior para registrar contextos e as relações desses conjuntos de coleções.

Existem iniciativas interessantes com o CRM, mas ainda são muito complexas e não foram muito absorvidas pelo corpo técnico das instituições. Não temos museus brasileiros trabalhando com ele, embora já exista projeto para a tradução do modelo para o português. O caso mais famoso do uso do modelo é do British Museum em Londres, o qual foi o único museu de grande porte que conseguiu fazer isso. Há certa dificuldade de passar da teoria para a prática, pois o alto nível de complexidade dos modelos dificulta a sua operacionalidade.

Alguns pesquisadores apontam que o CRM seria a peça que faltava para que de fato, se possam integrar modelos de dados nos universos distintos, o que sempre foi uma problemática. Pois, quando se conseguia programar alguma coisa por um lado não ficava adequado porque se usavam padrões estabelecidos de outra área e isso sempre gerava insatisfação no final.

A seguir focaremos em um padrão de metadados conhecido como Visual Resources Association (VRA Core), o qual indica ser o mais adequado para se trabalhar, quando se trata de obras de arte. Pois, o conjunto de elemento da versão VRA Core 4.0, por exemplo, fornece uma 
organização de categorias para a descrição da obra, da imagem que representa a obra bem como da coleção que faz parte a obra. Ou seja, três entidades: Obra, Imagem, Coleção e que se permite construir relações de registros Verges (2009).

O registro da obra, por exemplo, pode ser associado a uma ou mais imagens através do elemento relação. Da mesma forma que uma única imagem pode se relacionar a uma ou mais obras, quando, por exemplo, uma imagem de documentário é tomado de uma exposição e essa imagem retrata várias obras. Um registro de coleção pode ser usado para agregar trabalhos múltiplos ou vários registros de imagem.

\section{The Visual Resources Association (VRA Core): um breve estudo}

Associação de Recursos Visuais, conhecida como (VRA Core) ${ }^{10}$, é uma organização internacional destinada a profissionais que trabalham com instituições museológicas, ou que lidam com objetos de arte e patrimônios culturais.

Criada no ano de 1982 por bibliotecários membros do College Art Association (CAA), the South Eastern Art Conference (SECAC), the Art Libraries Society of North America (ARLIS/NA), e do Mid-America College Art Association (MACAA), a VRA preocupa-se com a formação de profissionais da imagem e desenvolve normas para o trabalho com obras de arte.

A VRA possui algumas versões, sendo a VRA 1.0 (1996) baseada no CDWA, VRA 2.0 (1996) que identificou a necessidade de padrões de conteúdos baseado no CCO, VRA 3.0 (2000) que possui os esquemas de metadados semelhantes ao esquema estrutural do Dublin Core, em sua simplicidade, número de elementos e uso de qualificadores. $E$ a versão atual VRA 4.0 que expressa em um esquema XML, a fim de apoiar a interoperabilidade e troca de registros VRA Core.

Tanto no Dublin Core quanto na VRA Core versão 3.0 os elementos e qualificadores são opcionais e podem ser repetidos em qualquer ordem.

O padrão possibilita que somente um recurso seja descrito dentro de um único conjunto de metadados e faz uma nítida distinção entre a obra e a imagem representada dessa obra. Prevê, também, que o conjunto descritivo de metadados dos registros possa ser criado para cada uma delas e lincado dentro de uma base de dados local.

A VRA 3.0 Record Type pode conter um dos dois valores, work ou image. Dessa forma, o registro de metadados representa uma obra ou uma imagem da obra. Embora esta distinção não faça tanto sentido em um contexto fora do museu, pode fornecer uma maneira clara de distinguirmos um objeto de arte que faz parte de um museu, de uma imagem documental que 0 objeto de arte retrata.

${ }^{10}$ Disponível em: <http://vraweb.org/>. Acesso em: 27 jul. 2016. 
A VRA Core 3.0 possui 17 esquemas de elementos, tecnicamente chamados de Categories. Assim como o Dublin Core, esta versão do VRA possui elementos estabelecidos, baseados no $X M L$, o que será demonstrado a seguir.

A figura 1 ilustra uma imagem digital da obra This is how it happened, do pintor espanhol Francisco Goya, feita com a técnica conhecida como "água forte", um tipo de gravura que faz parte da coleção da (MI,USA), University of Michigan Museum of Art.

Figura 1 - Obra de Francisco Goya -This is how it happened

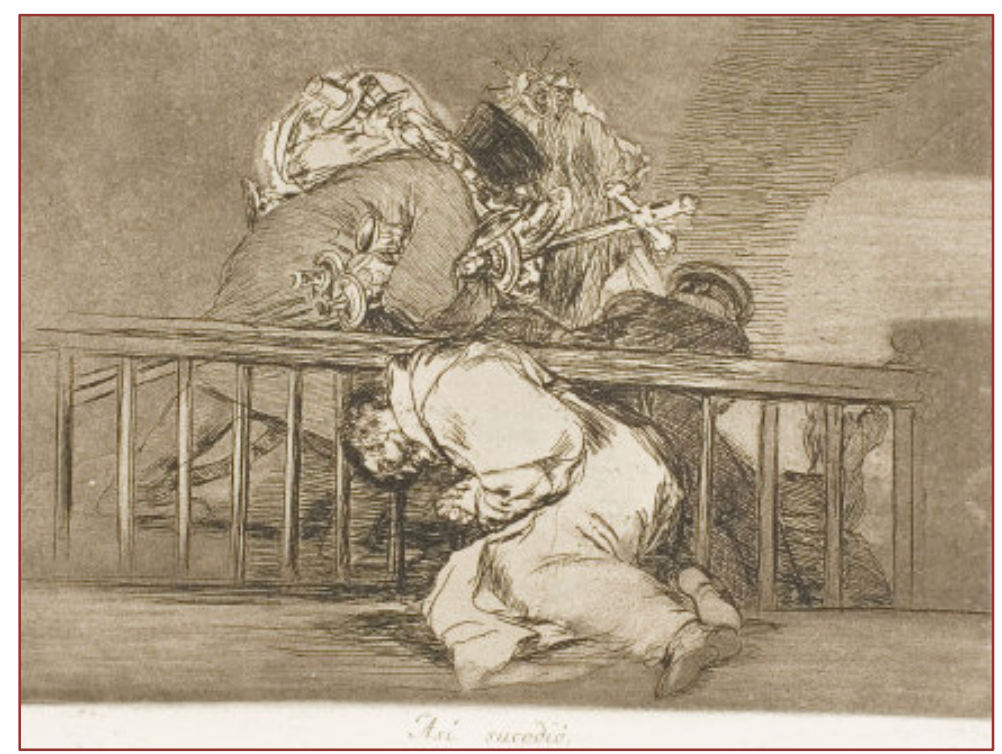

Fonte: Ann Arbor (MI,USA), University of Michigan Museum of Art.

Os Quadros 3 e 4 ilustram o conjunto de dados da obra This is how it happened nos seguintes aspectos: Work Record e Image Record, utilizando o padrão de metadados VRA Core 3.0.

Quadros 3 - conjunto de dados VRA core 3.0 (Work Record)

\begin{tabular}{r|l}
\hline Record Type & work \\
\hline Type & print \\
\hline Title & This is how it happened \\
\hline Title Variant & As Sucedi \\
\hline Measurements.Dimensions & $24.5 \times 35 \mathrm{~cm}$ \\
\hline Material. Medium & ink \\
\hline Technique & etching \\
\hline Technique & drypoint \\
\hline Creator. Personal Name & Francisco Jose de Goya y Lucientes \\
\hline Creator. Role & Printmaker
\end{tabular}




\begin{tabular}{r|l}
\hline Data. Creation & Ca.1810-1814 \\
\hline Location.Current Repository & Na Arbor (MI, USA)University of Michigan Museum of Art \\
\hline Location. Creation Site & Madri (Esp) \\
\hline Sty Number. Current Accession & $1977 / 2.15$ \\
\hline Culture & Spanish \\
\hline Subject & war \\
\hline Relation. Part of & Parto f Disasters of war \\
\hline Description & $\begin{array}{l}\text { This is how it happened is No.47(33) from the series "the Disaster } \\
\text { of War", } 4^{\text {th }} \text { edition, plates for the series ca.1810-14, 1820, } 4^{\text {th }} \\
\text { edition was published 1906. }\end{array}$ \\
\hline Rights & \multicolumn{1}{c}{ Weber family trust }
\end{tabular}

Source: Adapted from the VRA Core 3.0 "Compedium of Examples,

"http://www.vraweb.org/projects/vracore3/examples.html.data Standards Committee,

Visual Resources Association.

Fonte: MILLER (2004, p. 218).

Quadro 4 - Conjunto de dados VRA core 3.0 (Image Record)

\begin{tabular}{|c|c|}
\hline Record Type & image \\
\hline Type & digital \\
\hline Title & general view \\
\hline Measurements. Dimensions & 72 dpi \\
\hline Measurements. Format & jpeg \\
\hline Technique & scanning \\
\hline Creator & Fred Technician \\
\hline Data. Creation & 1999 \\
\hline Location. Current Repository & Na Arbor (MI, USA)University of Michigan Museum of Art \\
\hline $\begin{array}{r}\text { ID Number. Current } \\
\text { Accession }\end{array}$ & PCD5010-1611-1037-27 \\
\hline $\begin{array}{r}\text { ID Number. Current } \\
\text { Accession }\end{array}$ & $1977 / 2.15$ \\
\hline Description & $\begin{array}{l}\text { For more information, see } \\
\text { http://www.si.umich.edu/Art_History/demoareals/1977_2.15.html }\end{array}$ \\
\hline Source & University of Michigan Museum of Art \\
\hline Rights & University of Michigan Museum of Art \\
\hline
\end{tabular}

Source: see Table 8.2.

Fonte: MILLER (2004, p. 218). 
Diferentemente de softwares de bibliotecas, que possuem na sua maioria interfaces de saída mais voltadas e preocupadas com usuários, a interface de software de museus em geral é mais voltada para soluções internas. Geralmente, é tudo separado e há pouca informação disponível para usuários externos, até mesmo porque tradicionalmente a forma de extroversão do conhecimento da informação nos museus, de forma geral, são as exposições.

No entanto, vimos que temos que trabalhar em paralelo, pois a exposição é uma ferramenta de extroversão e as bases de dados de sistema de informação são tão importantes quanto as exposições. Os metadados são um dos componentes necessários para a integração de sistema de gestão e interoperabilidade em museus, como visto no exemplo acima possibilitado pelo VRA Core, o que deixou a interface e as informações das obras muito mais agradável e completa para os usuários.

A versão do padrão VRA Core 4.0 inclui o uso dos elementos e subelementos dos atributos sintáticos do XML. Esta nova versão possui 19 elementos essenciais, sendo o primeiro uma forma de invólucro, ou recipiente, utilizado para conter os outros 18 elementos. Alguns elementos possuem subelementos e atributos, e todos os elementos são opcionais e repetíveis, exceto para Work, Collection, ou Image, para os quais são necessários e não repetíveis.

O VRA Core 4.0 recomenda que os IDs de Work, por exemplo, sejam " $w$ "; de Image sejam " $\mathrm{i}$ " e de Collection sejam "c". Assim, tem-se um invólucro que contém todos os outros elementos em registros individuais, exemplo <image id = "i_002874">. Os elementos genéricos são chamados de Work, Collection ou Image, mas o nome real do elemento é um desses três termos, dependendo de qual dos três esteja sendo descrito no registro de metadados individual.

A área museológica, segundo Marcondes (2013), por não ter padrões de descrição consolidados, como em outras áreas, não possibilita que os museus de arte brasileiros façam uso de padrões estruturados de metadados. No entanto, gestores de museus ou profissionais que trabalham diretamente com a catalogação de obras de arte, e que necessitam deste tipo de ferramenta para facilitar o trabalho, encontram saídas em softwares privados, especializados em trabalhos de catalogação de obras de arte, mas que não possuem um padrão de metadados bem estabelecido ou compatível com o tipo de material com que o museu trabalha. A exemplo aqui no Brasil temos o DONATO.

Renomados museus de arte brasileiros, como Museu de Artes de São Paulo e a Pinacoteca do Estado de São Paulo, por exemplo, utilizam como padrão de informação o software gerenciador do banco de dados chamado Donato, versão atual 3.0, baseado no Thesaurus de Helena Dodd Ferrez e Maria Helena Bianchinni.

Este programa foi criado e adotado pelo Museu Nacional de Belas Artes para catalogação e gerenciamento de informações de seu acervo e desenvolvido para atender às necessidades de trabalho dos técnicos do MNBA, bem como de pesquisadores e visitantes Gemente (2015). O programa mantém características próprias para este Museu. 
A Pinacoteca utiliza o sistema Donato; ele foi desenvolvido para ser usado em museu de belas artes e possui problemas já resolvidos quando se trata de gravuras, esculturas, pinturas, gêneros mais tradicionais da arte. Mas quando se fala de fotografias, performances, bem como de recursos multimídias como videoarte e arte digital, por exemplo, já não serve; o sistema apresenta uma série de problemas porque simplesmente foi feito para um gênero mais tradicional, clássico, das belas artes e não para gêneros das artes contemporâneas.

A Pinacoteca, por exemplo, fez uma série de modificações no programa, como a gestão de direitos autorais, que é complicada para o museu. Há alguns campos da arte contemporânea que não existiam e não estavam no sistema original criado pelas belas artes. Em outras palavras, quando criarem uma nova versão do Donato, ou a Pinacoteca continua com a antiga para manter as mudanças que ela realizou, ou ela vai se adequar à nova, que não terá essas mudanças que ela implementou.

O uso do Donato em museus que possuem coleções de arte contemporânea acaba por modificar o padrão que foi criado para os gêneros das belas artes. Assim, acaba por desvirtuar a própria ferramenta. A proposta do Donato, de ser uma ferramenta para todos os tipos de museu, cai por terra, porque se cada museu o usa de um modo e tenta adaptar à sua maneira o padrão para se adequar à sua realidade, a tendência é que o Donato já não seja uma coisa só. E como se administra isso depois? Ele não foi feito para ser pensado como uma coisa dinâmica, esse é um dos grandes problemas. No entanto, propostas futuras da versão 4.0 segundo Gemente (2015) é a de interligar museus e usuários para o compartilhamento de informações entre pesquisadores e permitir aos técnicos das instituições integradas compartilharem documentos e pesquisas, tais como: bibliografias, laudos técnicos, solicitação de empréstimos etc.

O quadro 4 ilustra quais instituições brasileiras, como museus, galerias ou qualquer outro tipo de instituição que trabalhe com obras de arte e que utilizam o programa.

Quadro 4 - Instituições que utilizam o DONATO

\begin{tabular}{c|l}
\hline Bahia & Museu de Arte Sacra, Salvador \\
\hline \multirow{3}{*}{ Ceará } & $\begin{array}{l}\text { Memorial da Cultura Cearense, Fortaleza } \\
\text { Museu de Arte Contemporânea do Ceará, Fortaleza } \\
\text { Museu de Arte da Universidade Federal do Ceará, Fortaleza } \\
\text { Casa de José de Alencar, Fortaleza }\end{array}$ \\
\hline Distrito Federal & $\begin{array}{l}\text { Museu do Supremo Tribunal Federal, Brasília } \\
\text { Museu Vivo da Memória Candanga, Brasília }\end{array}$ \\
\hline \multirow{2}{*}{ Espírito Santo } & $\begin{array}{l}\text { Museu de Arte do Espírito Santo, Vitória } \\
\text { Galeria de Arte Espaço Universitário - UFES, Vitória } \\
\text { Diocese de Colatina, Colatina }\end{array}$ \\
\hline \multirow{2}{*}{ Maranhão } & $\begin{array}{l}\text { Museu Histórico e Artístico do Maranhão, São Luiz } \\
\text { Museu de Arte Sacra, São Luiz } \\
\text { Museu Cafua das Mercês, São Luiz } \\
\text { Museu de Artes Visuais, São Luiz }\end{array}$ \\
\hline \multirow{2}{*}{ Museu Regional de São João Del-Rei } \\
& $\begin{array}{l}\text { Museu do Garimpo, Tibagi } \\
\text { Museu Municipal de Carangola, Carangola }\end{array}$ \\
\hline
\end{tabular}




\begin{tabular}{|c|c|}
\hline & $\begin{array}{l}\text { Museu de Artes Murilo Mendes, Juiz de Fora } \\
\text { Museologia, Universidade Federal de Ouro Preto, Ouro Preto }\end{array}$ \\
\hline Pará & $\begin{array}{l}\text { Museu de Arte de Belém, MABE, Belém } \\
\text { Museu do Círio de Nazaré, Belém } \\
\text { Museu do Estado do Pará, MEP, Belém } \\
\text { Museu de Arte Sacra, Belém } \\
\text { Museu da Universidade Federal do Pará, Belém }\end{array}$ \\
\hline Paraíba & $\begin{array}{l}\text { Museu de Arte Assis Chateaubriand -MAAC, Campina Grande } \\
\text { Museu do Brejo Paraibano, Areia } \\
\text { Museu Casa de Pedro Américo, Areia } \\
\text { Museu Regional de Areia, Areia } \\
\text { Museu da Cultura Popular Paraibana, João Pessoa }\end{array}$ \\
\hline Paraná & $\begin{array}{l}\text { Museu Oscar Niemeyer, Curitiba } \\
\text { Museu Campus Gerais, Ponta Grossa } \\
\text { Casa da Memória de Carambeí, Carambeí }\end{array}$ \\
\hline Pernambuco & $\begin{array}{l}\text { Museu de Arte Moderna Aloísio Magalhães, Recife } \\
\text { Oficina Cerâmica Brennand, Recife } \\
\text { Museu do Estado de Pernambuco, Recife } \\
\text { Departamento de Arqueologia e Museologia (UFPE), Recife } \\
\text { Museu da Medicina de Pernambuco, Recife }\end{array}$ \\
\hline Rio de Janeiro & $\begin{array}{l}\text { Museu Nacional de Belas Artes } \\
\text { Museu Antônio Parreiras, Niterói } \\
\text { Fundação Eva Klabin Rappaport, Rio de Janeiro } \\
\text { Museu Histórico e Diplomático do Itamaraty, Rio de Janeiro } \\
\text { Museu de Imagens do Inconsciente, Rio de Janeiro } \\
\text { Museus Castro Maya, Rio de Janeiro } \\
\text { Museu Judaico do Rio de Janeiro, Rio de Janeiro } \\
\text { Museu de Arte Contemporânea de Niterói, Niterói } \\
\text { Instituto Fayga Ostrower, Rio de Janeiro } \\
\text { Museu Histórico Nacional } \\
\text { Museu de Arte Moderna de Resende, Resende } \\
\text { Ateliê Carlos Vergara, Rio de Janeiro } \\
\text { Museu de Arte Sacra, Angra dos Reis } \\
\text { Museu de Arqueologia de Itaipu, Niterói } \\
\text { Museu de Arte Religiosa e Tradicional, Cabo Frio }\end{array}$ \\
\hline Rio Grande do Sul & $\begin{array}{l}\text { Museu Joaquim José Felizardo, Porto Alegre } \\
\text { Museu Júlio de Castilhos, Porto Alegre } \\
\text { Museu Municipal, Caxias do Sul } \\
\text { Museu IPA (Instituto Porto Alegre) Porto Alegre } \\
\text { Museu das Missões, São Miguel das Missões } \\
\text { Fundação Vera Chaves Barcelos, Viamão } \\
\text { Museu de Artes Visuais Ruth Schneider, Passo Fundo } \\
\text { Museu Histórico, Farroupilha, Piratini } \\
\text { Museu Municipal Monsenhor Wolski, Santo Antonio das Missões } \\
\text { Museu Histórico Regional, Passo Fundo } \\
\text { Faculdade de Biblioteconomia e Comunicação (UFRGS), Porto } \\
\text { Alegre }\end{array}$ \\
\hline Santa Catarina & $\begin{array}{l}\text { Museu Hassis, Florianópolis } \\
\text { Museu Victor Meirelles, Florianópolis } \\
\text { Museu de Arte Contemporânea Luiz Henrique Schwanke, } \\
\text { Joinville }\end{array}$ \\
\hline São Paulo & $\begin{array}{l}\text { Pinacoteca do estado de São Paulo, São Paulo } \\
\text { Museu de Arte de São Paulo, São Paulo } \\
\text { Casa das Rosas, São Paulo } \\
\text { Casa Guilherme de Almeida, São Paulo } \\
\text { SESC-SP, São Paulo } \\
\text { Museu Penitenciário Paulista, São Paulo }\end{array}$ \\
\hline
\end{tabular}

Fonte: Disponível em: <http://www.mnba.gov.br/2_colecoes/simba/donato_0.htm>. Acesso em: 27 jul. 2016. 
Existem poucos estudos feitos em universidades brasileiras, por pesquisadores da área da Ciência da Informação, que procuram soluções adequadas para a interoperabilidade de informações museológicas. No entanto, pesquisadores que trabalham com informação, juntamente com profissionais da área museológica, estão pouco a pouco se adequando ao novo paradigma informacional e se esforçando com estudos e pesquisas para que haja uma melhora na interoperabilidade entre instituições museológicas que já fazem parte do sistema informacional global.

\section{Considerações finais}

Após a Internet ter se tornado o maior meio de comunicação da era da informação e pelo fato de grande parte dos recursos informacionais estarem passando por processo de digitalização, transformando-se em linguagem computacional de bits e bytes, possibilitando que os dados sejam manipulados por máquinas, todo processo de produção, representação, recuperação, acesso, processamento, transferência, visualização, armazenamento e preservação de dados está passando também por transformações e exige nova forma de fazer e pensar por parte dos profissionais que trabalham em unidades informacionais, nas mais variadas áreas, incluindo as que lidam com patrimônios culturais, como os museus.

É no ambiente Web que os museus estão disponibilizando, de forma digital, grande parte das informações sobre seu acervo. Mas, todas essas informações estão sendo disponibilizadas de forma não estruturada, dificultando a troca de dados, a comunicação e a interoperabilidade entre os sistemas de museus. Dessa forma, os padrões de metadados adequados para gerenciar toda a informação vinculada ao acervo se faz necessário para garantir o acesso e a recuperação da informação pelos usuários.

Existem alguns padrões de metadados específicos para ambientes que trabalham com obras de arte ou patrimônios culturais, como o VRA Core. No entanto, essa realidade só faz parte de alguns grandes museus em países mais desenvolvidos, como, por exemplo, o University of Michigan Museum of Art, nos Estados Unidos. No Brasil, os museus, em sua maioria, ainda não utilizam normas ou padrões de metadados que possibilitem uma melhora no acesso da informação pelo usuário nem a interoperabilidade entre sistemas de museus internacionais. Eles utilizam padrões internos, adaptados para cada museu, como o exemplo do programa de gerenciamento de banco de dados, DONATO, o qual permite a catalogação de obras de arte, e que é o mais utilizado entre os maiores museus do Brasil, mas que ainda não atende às expectativas de interoperabilidade e integração de sistemas.

Quase não há estudos no Brasil que se preocupem com essa questão. Estamos caminhando em passos lentos na elaboração de um ambiente museológico que acompanhe a tendência mundial de compartilhamento da informação, na qual já se constrói uma Web mais inteligente, rápida e compatível com os novos paradigmas informacionais. 


\section{Referências}

ALVES, R. C. V. Metadados como elementos do processo de catalogação. 2010. 132f. Tese (Doutorado em Ciência da Informação) - Faculdade de Filosofia Ciências, Universidade Estadual Paulista, Marília, 2010. Disponível em:

<http://www.athena.biblioteca.unesp.br/exlibris/bd/bma/33004110043P4 /2010/alves_rcv_dr_mar.pdf>. Acesso em: 20 jun. 2015.

ARAújo, C. A. A. Arquivologia, biblioteconomia, Museologia e Ciência da Informação: o diálogo possível. Brasilia, DF: Briquet de Lemos/Livros / São Paulo: Associação Brasileira de profissionais da Informação (ABRAINFO), 2014.

BACA, M. Introduction to Metadata: Pathways to Digital Information Getty Information Institute. 1998. Disponível em: http://www.getty.edu/research/conducting_research/standards/intrometa data/index.html. Acesso em: 10 Fev. 2016.

BEVILACQUA, G. M. F. Museu como serviço de informação. In: SEMINÁRIO SERVIÇO DE INFORMAÇÃO EM INSTITUIÇÕES CULTURAIS: em busca de conceitos, métodos e políticas de preservação, 2., 2014, São Paulo. Anais... São Paulo: Pinacoteca do Estado de São Paulo, 2014. p. 11-17.

COSTA, I. D. G.; ALMEIDA, M. C. B. Vocabulário de arte: ferramentas fundamentais no trabalho cooperativo em bibliotecas, museus e arquivos. In: SEMINÁRIO SERVIÇO DE INFORMAÇÃO EM MUSEU, 1., 2010, São Paulo. Anais... São Paulo: Pinacoteca do Estado de São Paulo, 2010. p. 89-101.

CRIPPA, G. A faceta humanística da Ciência da Informação: ordem e memória do/no museu. In: SEMINÁRIO SERVIÇO DE INFORMAÇÃO EM MUSEU, 1., 2010, São Paulo. Anais... São Paulo: Pinacoteca do Estado de São Paulo, 2010. p. 23-41

EUROPEANA. Europeana Data Model: Mapping Guidelines v. 2.2. Disponível em: <http://pro.europeana.eu/files/Europeana_Professional/Share_your_data/ Technical_requirements/EDM_Documentation/EDM_Mapping_Guidelines_v 2.2.pdf>. Acesso em: 5 Jul. 2015.

FERREZ, H. D. Documentação museológica: teoria para uma boa prática. In: FÓRUM NORDESTINO DE MUSEU, 4., Recife. Trabalhos apresentados... Recife: IBPC/Fundação Joaquim Nabuco, 1991. Disponível em: <http://www.crnti.edu.uy/02cursos/ferrez.doc>. Acesso em: $13 \mathrm{dez}$. 2015.

GEMENTE, G. Vinte anos de Donato: um breve histórico do Banco de Dados do Museu Nacional de Belas Artes. Rio de Janeiro: Museu Nacional de Belas

Artes, 2015.

Disponível em: 
<file:///C:/Users/Fabiano/Downloads/19-71-1-PB.pdf>. Acesso em: 22 maio 2016.

ISAAC, Antoine; CLAYPHAN, Robina; HASLHOFER, Bernhard - Europeana: moving to Linked Open Data. ISQ Information Standards Quarterly. v.42, n.2/3, 2012. Disponível em: http://www.niso.org/apps/group_public/download.php/9407/IP_Isaacetal_Europeana_isqv24no2-3.pdf. Acesso em: 06 Jul. 2015.

INTERNATIONAL COUNCIL OF MUSEUMS. The CIDOC Conceptual Reference Model. 2014. Disponível em: <http://www.cidoc-crm.org/>. Acesso em: 28 nov. 2014.

MARCONDES, C. H. Interligando acervos digitais na Web em arquivos, bibliotecas e museus. In: SEMINÁRIO INTERNACIONAL ARQUIVOS DE MUSEUS E PESQUISA: humanidades e interfaces digitais, 3., 2013, São Paulo. Anais... São Paulo: Grupo de Trabalho Arquivo de Museus e Pesquisas, 2013. p. 9-30.

MILLER, S. Metadata For Digital Collection: a how-to-do-it manual. New York; London: Neal-Schuman Publishers Inc., 2004.

NATIONAL INFORMATION STANDARDS ORGANIZATION (NISO). Understanding Metadata. Bethesda, MD: NISO Press, 2004. Disponível em:

<http://www.niso.org/publications/press/UnderstandingMetadata.pdf > .

Acesso em: set. 2015.

VERGES, J. VRA Core 4.0 na introduction. Boston, MA, 2009. Disponível em: <http://core.vraweb.org/pdfs/VRACore4SEI2009deVerges.pdf>. Acesso em: 21 Jun. 2016.

THE VISUAL RESOURCES ASSOCIATION - VRA Core. A data standard for the description of images and works of art and culture. Disponível em: <https://www.loc.gov/standards/vracore/schemas.html>. Acesso em: 27 jul. 2016.

YASSUDA, S. N. Documentação museológica: uma reflexão sobre o tratamento descritivo do objeto no Museu Paulista. 2009. 180f. Dissertação (Mestrado em Ciência da Informação) - Faculdade de Filosofia e Ciências, Universidade Estadual Paulista, Marília, 2009.

ZENG, M. L.; QIN, J. Metadata. New York: Neal-Schuman Publishers, 2008.

ZENG, M. L. Entendendo e utilizando o Linked Data em Bibliotecas, Arquivos e Museus (LAM). In: SEMINÁRIO INTERNACIONAL ARQUIVOS DE MUSEUS E PESQUISA: humanidades e interfaces digitais, 3., 2013, São Paulo. Anais... São Paulo: Grupo de Trabalho Arquivo de Museus e Pesquisas, 2013. p. 93-109. 